\title{
Thermal Expansion and Phase Transformations of Low-Expanding Cobalt-Iron-Chromium Alloys
}

\author{
Peter Hidnert and Richard K. Kirby
}

\begin{abstract}
Coefficients of linear thermal expansion of some cobalt-iron-chromium alloys are reported for various temperature ranges between $-65^{\circ}$ and $+800^{\circ} \mathrm{C}$, and the effects due to temperature, chemical composition, heat treatment, etc., were determined. Some of the alloys investigated have coefficients of expansion less than those for fused quartz and ordinary invar between $-65^{\circ}$ and $+60^{\circ} \mathrm{C}$. Some of the low-expanding cobalt-iron-chromium alloys have $\gamma \rightarrow \alpha$ transformations on cooling to low temperatures, and the resulting $\alpha$-phase reverted to $\gamma$ on heating to high temperatures. $\mathrm{Ar}_{3}$ temperatures were observed as high as $-10^{\circ} \mathrm{C}$ and $\mathrm{Ac}_{1}$ temperatures at about $600^{\circ} \mathrm{C}$. The effects of various heat treatments from $-196^{\circ}$ to $+1,000^{\circ} \mathrm{C}$ on the transformations were investigated, and the resulting changes of thermal expansion were correlated with the structure of these alloys.
\end{abstract}

\section{Introduction}

In 1934 , Masumoto $[1]^{1}$ reported the results of his investigation on the linear thermal expansion of cobalt-iron-chromium alloys containing more than 49 percent of cobalt and 5 to 20 percent of chromium. These alloys were prepared by mixing suitable proportions of electrolytic cobalt, electrolytic iron, and chromium (with $2.7 \%$ of impurities) and melting these metals in alumina crucibles in a hydrogen atmosphere. The melts were cast in iron molds 26 $\mathrm{cm}$ long and $0.6 \mathrm{~cm}$ in diameter. Samples $10 \mathrm{~cm}$ in length were cut from the ingots and heated for $1 \mathrm{hr}$ at $1,000^{\circ} \mathrm{C}$ in a vacuum furnace and then cooled slowly in the furnace. The chemical compositions indicated by Masumoto for these alloys were not obtained by chemical analyses [2] but were computed by him from the initial charges.

Some of these alloys investigated by Masumoto have very small coefficients of expansion. For example, the coefficient of expansion of an alloy with a nominal composition of iron 36.5 percent and chromium 9.5 percent is $0.1 \times 10^{-6} / \mathrm{deg} \mathrm{C}$ between $20^{\circ}$ and $60^{\circ} \mathrm{C}$. This expansion coefficient is less than those of such well-known low-expanding materials as fused quartz [3] and the usual grade of invar [4]. The investigation of these low-expanding cobalt-iron-chromimum alloys was made over the range from liquid-air temperature to about $+250^{\circ} \mathrm{C}$. Masumoto stated that the observations above room temperature were taken during heating, as the expansion was almost reverisble for heating and cooling, and the observations below room temperature were taken during cooling.

In his paper Masumoto also reported results of investigations on some magnetic, electrical, and corrosion-resisting ${ }^{2}$ properties of one of these lowexpanding alloys. Data on Young's modulus and the temperature coefficient of Young's modulus for various cobalt-iron-chromium alloys were reported by Masumoto and Saito [5].

${ }^{1}$ Figures in brackets indicate the literature references at the end of this paper. 2 This alloy resisted indicate the literature references at the end of this paper. greater extent than invar, and therefore Masumoto named it "Stainless-Invar."
During World War II, Eastman Kodak Co., Rochester, N. Y., obtained a Government contract from the Office of Scientific Research and Development to produce a low-expanding alloy having a coefficient of expansion less than that of a good grade of invar at atmospheric temperatures. After a search of the literature and some preliminary tests with the cooperation of other companies, Eastman Kodak Co. authorized the Unexcelled Manufacturing Co., Cambridge, Mass., to prepare some low-expanding cobalt-iron-chromium alloys similar to those investigated by Masumoto. Peter Hidnert and Wilmer Souder of the National Bureau of Standards made determinations of linear thermal expansion on a number of these alloys over the range from $-65^{\circ}$ to $+60^{\circ} \mathrm{C}$. A restricted report of the results of this work was prepared by Edgar D. Seymour of Eastman Kodak Co. for the Office of Scientific Research and Development. The following summary was taken from an unclassified extract of the report:

From a search of the literature for a low-expanding metal, Masumoto's cobalt, iron, and chromium alloy gave the most promise, if the melting, heat treatment, and analysis problems could be solved. We believe these problems have been solved and the technique has been perfected so that it is possible to specify the composition to $0.1 \%$ in chromium and iron. An alloy has been made with a coefficient of expansion of less than $\pm 0.5 \times 10^{-6} /{ }^{\circ} \mathrm{C}$ over the range from $-65^{\circ}$ to $+60^{\circ} \mathrm{C}$ with the coefficient nearly zero from $20^{\circ}$ to $45^{\circ} \mathrm{C}$ and also in the region of $-35^{\circ} \mathrm{C}$. This is from two to three times better than invar obtainable on the market.

The data reported by Seymour on thermal expansion of cobalt-iron-chromium alloys is included in the present paper. Additional data by the present authors on these alloys and other cobalt-ironchromium alloys are given for various temperatures between $-65^{\circ}$ and $+950^{\circ} \mathrm{C}$.

\section{Alloys Investigated}

The cobalt-iron-chromium alloys listed in table 1 were prepared by John Wulff of the Unexcelled Manufacturing Co., Cambridge, Mass. The alloys were made from cobalt, iron, and chromium powders. A master alloy of cobalt and chromium was first 
TABLE 1. Coefficients of linear thermal

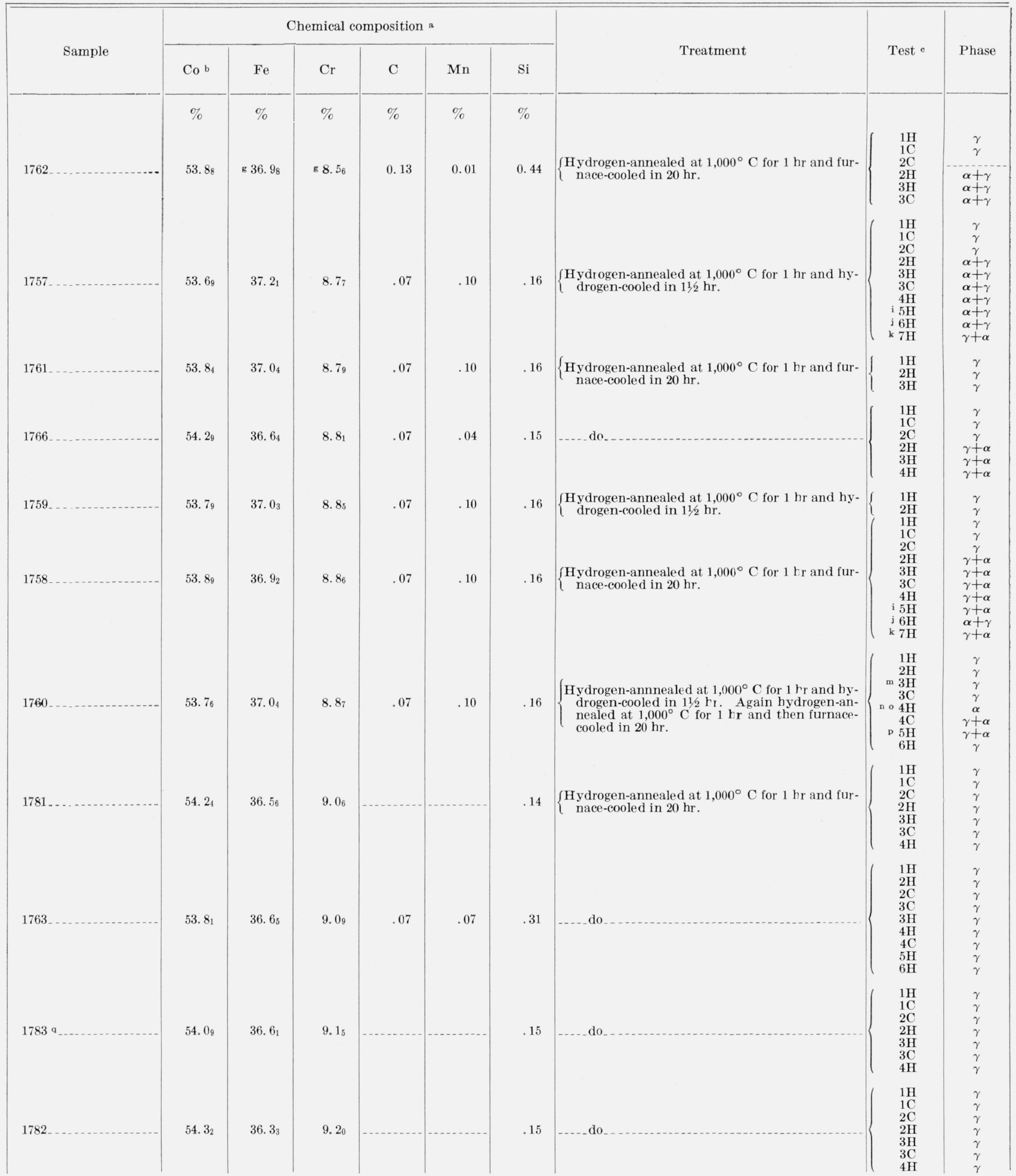




\begin{tabular}{|c|c|c|c|c|c|c|c|c|c|c|c|c|c|c|}
\hline \multicolumn{14}{|c|}{ Average coefficients of expansion per deg C } & \multirow{2}{*}{$\begin{array}{l}\text { Residual } \\
\text { change in } \\
\text { length after } \\
\text { each test } f\end{array}$} \\
\hline $\begin{array}{r}-60^{\circ} \text { to } \\
-20^{\circ} \mathrm{C} \text { d }\end{array}$ & $\begin{array}{l}-20^{\circ} \text { to } \\
+20^{\circ} \mathrm{C}\end{array}$ & $\begin{array}{l}-60^{\circ} \text { to } \\
+20^{\circ} \mathrm{C}^{\mathrm{e}}\end{array}$ & $\begin{array}{l}20^{\circ} \text { to } \\
60^{\circ} \mathrm{C}\end{array}$ & $\begin{array}{l}60^{\circ} \text { to } \\
100^{\circ} \mathrm{C}\end{array}$ & $\begin{array}{l}100^{\circ} \text { to } \\
150^{\circ} \mathrm{C}\end{array}$ & $\begin{array}{l}150^{\circ} \text { to } \\
200^{\circ} \mathrm{C}\end{array}$ & $200^{\circ}$ to $250^{\circ} \mathrm{C}$ & $\begin{array}{l}250^{\circ} \text { to } \\
300^{\circ} \mathrm{C}\end{array}$ & $\begin{array}{l}20^{\circ} \text { to } \\
100^{\circ} \mathrm{C}\end{array}$ & $\begin{array}{l}20^{\circ} \text { to } \\
150^{\circ} \mathrm{C}\end{array}$ & $\begin{array}{l}20^{\circ} \text { to } \\
200^{\circ} \mathrm{C}\end{array}$ & $\begin{array}{l}20^{\circ} \text { to } \\
250^{\circ} \mathrm{C}\end{array}$ & $\begin{array}{l}20^{\circ} \text { to } \\
300^{\circ} \mathrm{C}\end{array}$ & \\
\hline$\times 10^{-6}$ & $\times 10^{-6}$ & $\times 10^{-6}$ & $\times 10^{-6}$ & $\times 10^{-6}$ & $\times 10^{-6}$ & $\times 10^{-6}$ & $\times 10^{-6}$ & $\times 10^{-6}$ & $\times 10^{-6}$ & $\times 10^{-6}$ & $\times 10^{-6}$ & $\times 10^{-6}$ & $\times 10^{-6}$ & $\%$ \\
\hline & -.....- & & 1. 1 & ..... &..-- & -.... & $-\ldots$ & -...- & ..... & -.... & ..... & ... & .... & 0.000 \\
\hline$\left(\mathrm{T}_{t}=-67^{\circ}\right)$ & (n..... & $\left(\mathrm{Ar}_{3}=-10^{\circ}\right)$ & 1. 2 & 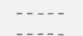 & -.... & -.... & -.... & -...- & -.... & -.... & (n.... & (n) & 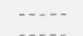 & 0.000 \\
\hline $\begin{array}{r}\left(1_{\mathrm{L}}=-6.7\right. \\
6.7\end{array}$ & 4.8 & $\left(A r_{3}=-10\right)$ & $\cdots$ & - & - & n.... & - & - & (n. & - n. & $\cdots$ & - & $\ldots$ & +.228 \\
\hline (n. & -...... & - n & 5. 2 & $\ldots$ & ..... & -.... & ..... & ..... & ..... & ..... & -.... & -.... & $-\ldots$ & -.002 \\
\hline$\cdots$ & -.... & $\ldots$ & 5. 6 & ..... & ..... & -.... & $\ldots$ & ..... & $\ldots$ & $\ldots$ & $\cdots$ & ....... & $\ldots$ & .00 \\
\hline ... & ...... & $\ldots$ & .8 & $\ldots$ & -... & -.... & -.... & -.... & ..... & -.... & -.... & -.... & $\ldots$ & .000 \\
\hline$\left(\mathrm{T}_{\mathrm{L}}=-64^{\circ}\right)$ & h. 1 & $\left(\mathrm{Ar}_{3}=-35^{\circ}\right)$ & $\begin{array}{r}.8 \\
-.-.\end{array}$ & $\ldots$ & ..... & - & - & $\ldots$ & $\ldots$ & $\ldots$ & $\ldots$ & - & 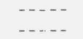 & +170 \\
\hline 6. 6 & 3.8 & 5. 2 & 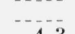 & $\ldots$ & $\ldots$ & ..... & .... & $\ldots$ & $\ldots$ & .... & - & .... & - & +.170 \\
\hline -... & ..... & -... & $\begin{array}{l}\text { 4. } 3 \\
\text { 4. } 5\end{array}$ & ..... & ..... & -... & -... & -.... & .... & ..... & -... & -... & -... & -.001 \\
\hline . & ...... & $\cdots$ & $\begin{array}{l}\text { 4. } 6 \\
\text { 4. } 6\end{array}$ & 5.4 & 7.7 & 10.6 & -.... & -..... & 5.0 & 6.0 & 7.3 & - & 9.7 & +.003 \\
\hline - & - & - & 4. 4 & 5. 4 & 7.8 & 10. 7 & - & - n & 4.9 & 6.0 & 7. 3 & - & 9.7 & +.001 \\
\hline$\ldots$ & ...... & - & 4. 3 & 5.8 & 7. 8 & 11.1 & ..... & $\ldots$ & 5. 1 & 6.1 & 7.5 & $\ldots$ & 9.7 & .000 \\
\hline -... & -..... & - & 2.6 & 4.0 & ...... & -.... & -.... & -.... & 3.3 & -.... & 6.7 & $\ldots$ & $\ldots$ & -.001 \\
\hline & - . . & & .2 & 1. 4 & 5. 2 & 10.9 & $\ldots$ & $\ldots$ & .8 & 2.5 & 4. 8 & $\ldots$ & 8.5 & -.001 \\
\hline .... & ...... & & -.2 & 1. 4 & ..... & ..... & $\ldots$ & .... & .6 & ..... & $\ldots$ & $\ldots$ & $\ldots$. & .000 \\
\hline -..- & ...... & -.......... & -.2 & 1.6 & ..... & -.... & -.... & $\ldots$ & .7 & $\ldots$ & ..... & ..... & ..... & .000 \\
\hline -. & & & 1. 4 & $\ldots$ & ..... & $\ldots$ & $\ldots$ & ..... & ...... & $\ldots \ldots$ & ...... & $\ldots$ & .....- & .000 \\
\hline$\left(\mathrm{T}_{\mathrm{L}}=-65^{\circ}\right)$ & 7 & $\left(\mathrm{Ar}_{3}=-40^{\circ}\right)$ & $\begin{array}{r}1.4 \\
-\ldots\end{array}$ & 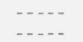 & ..... & $\ldots$ & $\ldots$ & $\ldots$ & $\ldots$ & - & - & -.... & ..... & .000 \\
\hline $\begin{array}{ll}2.5 \\
2.5\end{array}$ & 1. 0 & 1.7 & $\cdots$ & $\cdots$ & ne & - nes & - & - & $\cdots$ & - ne & 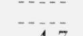 & $\ldots$ & $\cdots$ & +. 019 \\
\hline (..... & ....... & 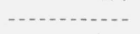 & .8 & 2.0 & 4. 9 & 9.8 & $\ldots$ & $\ldots$ & 1. 4 & 2.8 & 4. 7 & $\ldots$ & 8.3 & -.001 \\
\hline$\cdots$ & -..... & - & 1.0 & 1.9 & $\ldots$ & $\ldots$ & -... & ..... & 1.5 & $\ldots$ & -.... & -.... & ..... & .000 \\
\hline & ...... & & .4 & 1. 6 & 4. 6 & 9.9 & 14.4 & 15.4 & 1. 0 & 2. 4 & 4. 4 & 6. 6 & 8. 2 & -.002 \\
\hline (n.... & ...... & & .2 & 1.8 & ...... & ..... & .... & ..... & 1.0 & ..... & ..... & $\ldots$ & ..... & .000 \\
\hline (n...... & ....... & (........ & .2 & $\ldots$. & $\ldots$ & $\cdots$ & $\ldots$ & -... & $\cdots$ & $\ldots$ & ..... & $\ldots$ & ..... & -.001 \\
\hline$\left(\mathrm{T}_{\mathrm{L}}=-64^{\circ}\right)$ & 2 & $\left(\mathrm{Ar}_{3}=-40^{\circ}\right)$ & $\begin{array}{r}.4 \\
\ldots .\end{array}$ & $\cdots$ & $\cdots$ & $\ldots$ & $\ldots$ & $\ldots$ & - . . & $\ldots$ & $\ldots$ & $\cdots$ & $\ldots$ & +146 \\
\hline 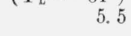 & 2. 8 & 4. 2 & -1 & ..... & ..... & -.... & (n.... & - & - & - & -... & - & - & +.146 \\
\hline (n....... & ...... & .... & 3.4 & $\ldots$ & $\ldots$ & -.... & ...... & $\ldots$ & $\ldots$ & ..... & ..... & ..... & ..... & -.001 \\
\hline . & - & . & $\begin{array}{l}3.7 \\
3.7\end{array}$ & 4.7 & 7.3 & 11.2 & $\cdots$ & $\cdots$ & 4.2 & 5.4 & 7.0 & $\ldots$ & 9.7 & +.001 \\
\hline . & - & (n) & 3.7 & 4.8 & 7.1 & 11. 6 & 13. 7 & 14.9 & 4. 2 & 5. 3 & 7. 1 & 8.5 & 9.6 & .000 \\
\hline - & - & - & 5.5 & 6.4 & 8.5 & 11. 3 & 13. 4 & 14. 6 & 5. 9 & 6. 9 & 8. 1 & 9. 3 & 10.2 & +.001 \\
\hline ............ & ....... & - & 2.4 & 4. 0 & $\ldots$ & $\ldots$ & $\ldots$ & $\ldots$ & 3.2 & $\ldots$ & 6.7 & $\ldots$ & ...... & .000 \\
\hline .... & - n.... & $\ldots$ & .3 & 2. 0 & 5.0 & 10.7 & 14. 6 & 15. 7 & 1. 1 & 2. 6 & 4. 9 & 7.0 & 8.5 & -.001 \\
\hline .... & -..... & .... & .3 & 1. 8 & & & & & 1. 1 & & & & & .000 \\
\hline (n........ & ...... & - & .6 & 2.0 & 5. 6 & 10.1 & 13.9 & 15. 6 & 1. 3 & 3.0 & 4. 9 & 6.9 & 8.4 & -.011 \\
\hline$\cdots$ & (n) & - & 5.9 & 7.4 & $\cdots$ & -.... & $\cdots$ & -.... & 6.6 & $\cdots$ & $\begin{array}{l}4.6 \\
8.7\end{array}$ & $\ldots$ & $\begin{array}{r}8.2 \\
10.6\end{array}$ & \\
\hline . & . & - & $\begin{array}{l}5.9 \\
4.5\end{array}$ & $\begin{array}{l}7.4 \\
7.3\end{array}$ & ...... & $\ldots$ & -... & -.... & $\begin{array}{l}6.6 \\
5.9\end{array}$ & -.... & $\begin{array}{l}8.7 \\
9.3\end{array}$ & ..... & $\begin{array}{l}10.6 \\
11.3\end{array}$ & -.276 \\
\hline - & - & - & 4. 4 & 7. 2 & n.... & $\ldots$ & (n..... & n...... & 5. 8 & (n...... & $\begin{array}{r}\$ .0 \\
\ldots\end{array}$ & -.... & 11. 4 & \\
\hline ....... & -..... & . . & .6 & 2.1 & $\ldots$ & $\ldots$ & $\ldots$ & $\ldots$ & 1. 3 & $\ldots$ & $\ldots$ & $\ldots$ & $\ldots$ & .000 \\
\hline -..- & ....... & - . & .6 & ..... & $\ldots$ & ..... & ..... & -.... & ...... & $-\ldots$ & $\ldots$ & $\ldots$ & -.... & 000 \\
\hline & & & .6 & $\ldots$ & ..... & .... & ..... & ..... & ..... & $\ldots$ & ..... & $\ldots$ & -.... & .000 \\
\hline$\therefore 4$ & .3 & .4 & ...... & $\ldots$ & -.... & ...... & $\ldots$ & ..... & ...... & $\ldots$ & -.... & $\ldots$ & $\ldots$ & +.004 \\
\hline 1. 2 & .5 & .8 & 8 & $\cdots$ & ..... & $\ldots$ & $\cdots$ & $\cdots$ & $\cdots$ & $\cdots$ & $\cdots$ & $\cdots$ & $\ldots$. & 1.50 \\
\hline - & ....... & (n. & $\begin{array}{l}.8 \\
.7\end{array}$ & ..... & -.... & -.... & -..... & ..... & ...... & $\ldots$ & $\ldots$ & $\ldots$ & ...... & +.001 \\
\hline (n) & - & (n) & .8 & 2.5 & 5.6 & 10.9 & (n.... & (n) & 1.7 & 3.2 & 5. 3 & ..... & 8.8 & -.001 \\
\hline & -..... & (n... & 1. 1 & ..... & ..... & ..... & $\ldots$ & $\ldots$ & -.... & $\ldots$ & $\ldots$ & $\ldots$ & ..... & .000 \\
\hline (n.... & ....... & $\ldots$ & 1. 2 & $\ldots$ & ...... & $\ldots$ & -.... & -..... & $\ldots$ & $\ldots$ & $\ldots$ & ..... & $\ldots$ & .000 \\
\hline-.2 & -1 & -2 & $\begin{array}{r}1.3 \\
-\end{array}$ & -.... & ...... & -.... & (n.... & -.... & ......... & (..... & (n.... & (n.... & ..... & + \\
\hline 0 & .0 & .0 & $\ldots$ & $\ldots$ & $\ldots$ & $\ldots$ & $\ldots$ & $\ldots$ & n...... & $\ldots$ & $\ldots$ & $\ldots$ & $\ldots$ & +.002 \\
\hline ... & ...... & (n) & 1. 3 & $\ldots$ & -...- & -...- & $\ldots$ & $\ldots$ & $\ldots$. & $\ldots$ & ..... & .... & ..... & .000 \\
\hline - & . & (n.... & $\begin{array}{l}\text { 1. } 2 \\
\text { 1. } 4\end{array}$ & 4.2 & ...... & (..... & -..... & n.... & 2.8 & $\ldots$ & -..... & & & .000 \\
\hline ............ & - & 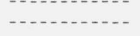 & $\ldots .$. & $\ldots .$. & 8.2 & 13.0 & 15. 2 & 16. 0 & 2. 8 & 4. 8 & 7.1 & 8. 9 & 10.1 & -.001 \\
\hline - & -..... & - & .6 & -.. & -.... & $\ldots$ & -...- & - . - & $\ldots$ & $\ldots$ & -...- & -.... & -.... & 000 \\
\hline - & $\cdots$ & - & .6 & -... & -.... & -... & -...- & .... & ..... & ..... & -... & -.... & -.... & \\
\hline+.1 & $\begin{array}{l}-.4 \\
-.1\end{array}$ & $\begin{array}{l}-.2 \\
+3\end{array}$ & -.... & $\ldots$ & $\ldots$ & $\ldots$ & $\ldots$ & $\ldots$ & $\ldots$ & $\ldots$ & $\ldots$ & ..... & ....- & +.004 \\
\hline $\begin{array}{c}.0 \\
-.-\end{array}$ & -.1 & $\begin{array}{r}\text { †. } \\
.\end{array}$ & .7 & $\cdots$ & 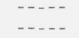 & - & $\ldots$ & (n.... & (n.... & $\ldots$ & 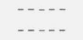 & $\ldots$ & -.... & -001 \\
\hline (n) & (n) & - & $\begin{array}{r}.8 \\
1.0\end{array}$ & 2.7 & 6.3 & 11.9 & 15.0 & 16.2 & 1.8 & 3.6 & 5.9 & 7.9 & 9.3 & -.001 \\
\hline & & & & & & & & & & & & & & \\
\hline$\cdots$ & - & $\cdots$ & $\begin{array}{l}1.0 \\
1.0\end{array}$ & -.... & -... & -.... & -.... & $-\cdots$ & -.... & -.... & -.... & $\ldots$ & ..... & .000 \\
\hline 8 & +.5 & .7 & $\ldots \ldots$ & $\ldots$ & $\ldots$ & $\ldots$ & $-\ldots$ & $-\ldots$ & $-\ldots$ & $\ldots$ & $\ldots$ & $\ldots$ & $\ldots$ & 000 \\
\hline 1. 0 & .4 & .7 & $\ldots$ & $\ldots$ & $\ldots$ & $\ldots$ & $\ldots$. & $\ldots$. & $\ldots$ & $\ldots$. & $\ldots$ & ..... & ..... & .000 \\
\hline$\ldots$ & (n...... & (n. & 1. 0 & $\ldots$ & ..... & -.... & $\ldots$ & $\ldots$ & $\ldots$ & ..... & ..... & -... & $\ldots$ & .000 \\
\hline - & (n...... & - & 1. 0 & 2.7 & 6.2 & 11.4 & 14.6 & 15.8 & 1.9 & 3.6 & 5.7 & 7.7 & 9.1 & -.001 \\
\hline
\end{tabular}


Taвle 1. Coefficients of linear thermal

\begin{tabular}{|c|c|c|c|c|c|c|c|c|c|}
\hline \multirow{2}{*}{ Sample } & \multicolumn{6}{|c|}{ Chemical composition s } & \multirow{2}{*}{ Treatment } & \multirow{2}{*}{ Test c } & \multirow{2}{*}{ Phase } \\
\hline & $\mathrm{Co} b$ & $\mathrm{Fe}$ & $\mathrm{Cr}$ & $\mathrm{C}$ & Mn & $\mathrm{Si}$ & & & \\
\hline $1767 \ldots$ & 53. $4_{0}$ & $\%$ & 9. 30 & $\%$ & $\%$ & $\%$ & $\left\{\begin{array}{c}\text { Hydrogen-annealed at } 1,000^{\circ} \mathrm{C} \text { for } 1 \mathrm{hr} \text { and fur- } \\
\text { nace-cooled in } 20 \mathrm{hr} \text {. }\end{array}\right.$ & $\begin{array}{l}1 \mathrm{H} \\
1 \mathrm{C} \\
2 \mathrm{C} \\
2 \mathrm{H} \\
3 \mathrm{H} \\
3 \mathrm{C} \\
4 \mathrm{H}\end{array}$ & $\begin{array}{l}\gamma \\
\gamma \\
\gamma \\
\gamma \\
\gamma \\
\gamma \\
\gamma\end{array}$ \\
\hline $1770 \ldots$ & 53. $5_{0}$ & 36. $7_{0}$ & 9. 36 & .08 & .07 & .29 & _... do . . & $1 \mathrm{H}$ & $\gamma$ \\
\hline $1772 \ldots$ & 53. 53 & $36.6_{1}$ & 9. $4_{3}$ & .08 & .07 & .28 & $\ldots$ do & $\begin{array}{l}1 \mathrm{H} \\
1 \mathrm{C} \\
2 \mathrm{C} \\
2 \mathrm{H} \\
3 \mathrm{H} \\
3 \mathrm{C} \\
4 \mathrm{H}\end{array}$ & $\begin{array}{l}\gamma \\
\gamma \\
\gamma \\
\gamma \\
\gamma \\
\gamma \\
\gamma\end{array}$ \\
\hline $1769 \ldots$ & 53. 56 & 36. 53 & 9. $\Sigma_{6}$ & .06 & .07 & .22 & $\ldots$ do .... & $\begin{array}{l}1 \mathrm{H} \\
1 \mathrm{C} \\
2 \mathrm{C} \\
2 \mathrm{H} \\
3 \mathrm{H} \\
3 \mathrm{C} \\
4 \mathrm{H}\end{array}$ & $\begin{array}{l}\gamma \\
\gamma \\
\gamma \\
\gamma \\
\gamma \\
\gamma \\
\gamma\end{array}$ \\
\hline $1764^{\mathrm{r}} \ldots \ldots$ & 53. $3_{0}$ & 36. 63 & 9. 57 & .09 & .08 & .33 & ..... do & $\begin{array}{l}1 \mathrm{H} \\
2 \mathrm{H}\end{array}$ & $\begin{array}{l}\gamma \\
\gamma\end{array}$ \\
\hline $1765 \mathrm{~A}^{8} \ldots$ & 53. $1_{7}$ & 36.78 & 9. 57 & & & & $\ldots$ do .... & $\begin{array}{l}1 \mathrm{H} \\
1 \mathrm{C} \\
2 \mathrm{C} \\
2 \mathrm{H} \\
3 \mathrm{H} \\
3 \mathrm{C} \\
4 \mathrm{H}\end{array}$ & $\begin{array}{l}\gamma \\
\gamma \\
\gamma \\
\gamma \\
\gamma \\
\gamma \\
\gamma\end{array}$ \\
\hline $1773 \ldots$ & 53. $9_{2}$ & 36. $2_{2}$ & 9. $6_{7}$ & .07 & .07 & .05 & do _. & $\begin{array}{l}1 \mathrm{H} \\
1 \mathrm{C} \\
2 \mathrm{C} \\
2 \mathrm{H} \\
3 \mathrm{H} \\
3 \mathrm{C} \\
4 \mathrm{H}\end{array}$ & $\begin{array}{l}\gamma \\
\gamma \\
\gamma \\
\gamma \\
\gamma \\
\gamma \\
\gamma\end{array}$ \\
\hline $1765 \ldots$ & 53. $1_{4}$ & 36. $5_{1}$ & 9. 87 & .11 & .10 & .27 & .... do & $\begin{array}{c}\mathrm{t} 1 \mathrm{H} \\
1 \mathrm{C} \\
2 \mathrm{C} \\
2 \mathrm{H} \\
3 \mathrm{H} \\
3 \mathrm{C} \\
4 \mathrm{H}\end{array}$ & $\begin{array}{l}\gamma \\
\gamma \\
\gamma \\
\gamma \\
\gamma \\
\gamma \\
\gamma\end{array}$ \\
\hline
\end{tabular}

s Feand Cr by J. I. Shultz and R. K. Bell of the National Bureau of Standards; C, Mn, and Si by Walter M. Saunders, Jr. Data on two samples (1752 and 1753) were discarded on account of segregation.

b By difference.

c $\mathrm{H}$ indicates that the coefficients of expansion were obtained on heating and $\mathrm{C}$ indicates that they were obtained on cooling.

d $T_{L}$ indicates the lowest observed temperature during the test.

- Ars indicates the initial temperature of the $\gamma \rightarrow \alpha$ transformation as evidenced by sudden expansion on cooling.

y sudden expansion on cooling. The plus sign indicates an increase in length and the minus sign a decrease in The plus
length

s A pproximate

From $-30^{\circ}$ to $+20^{\circ} \mathrm{C}$

i Before this test, cooled to $-80^{\circ} \mathrm{C}$.

made in a high-frequency furnace. Using this as stock material, a series of runs was then made with various iron additions to determine by chemical analyses the losses met with in melting the same weight of material each time. In order to compensate for these losses, desired amounts of the metal powders were added to the remelts before casting. Before pouring, the melts were deoxidized with definite amounts of calcium-silicon alloy. The cast rods $(3 / 8$-in. diam.) were then heat-treated.

Table 1 gives information about the heat treatments and the chemical compositions of the cobaltiron-chromium samples investigated. The chemical j Before this test, cooled to $-196^{\circ} \mathrm{C}$.

$\mathrm{k}$ Before this test, hydrogen-annealed at $850^{\circ} \mathrm{C}$ for $10 \mathrm{hr}$ and at $1,000^{\circ} \mathrm{C}$ for 1 $\mathrm{hr}$, and furnace-cooled in $22 \mathrm{hr}$.

1 From $20^{\circ}$ to $50^{\circ} \mathrm{C}$

$m$ During this test, heated to $700^{\circ} \mathrm{C}$ (see table 2)

n Before this test, cooled to $-190^{\circ} \mathrm{C}$.

- During this test, heated to $800^{\circ} \mathrm{C}$ (see table 2).

D During this test, heated to $950^{\circ} \mathbf{C}$ (see table 2 for coefficients of expansion to $\left.800^{\circ} \mathrm{C}\right)$.

a On cooling a duplicate sample rapidly, an Ar3 transformation was observed at about $-80^{\circ} \mathrm{C}$

$\mathrm{r}$ No Ars transformation was observed on cooling a duplicate sample to $-196^{\circ} \mathrm{C}$.

$\mathrm{r}$ No Ar3 transformation was observed on coolin
This sample is not a duplicate of sample 1765 .

This sample is not a duplicate of sample 1765 .

analyses were generally made on pieces cut from the ends of the samples. The iron contents varied from $36.2_{2}$ to $37.2_{1}$ percent, and the chromium contents from $8.5_{6}$ to $9.8_{7}$ percent by weight.

\section{Apparatus}

The micrometric thermal-expansion apparatus described by Hidnert and Souder [6] was used for the determinations of linear thermal expansion of the cobalt-iron-chromium samples. Each sample was about $300 \mathrm{~mm}$ long. 
expansion of cobalt-iron-chromium alloys-Continued

\begin{tabular}{|c|c|c|c|c|c|c|c|c|c|c|c|c|c|c|}
\hline \multicolumn{14}{|c|}{ A verage coefficients of expansion per deg $C$} & \multirow{2}{*}{$\begin{array}{l}\text { Residual } \\
\text { change in } \\
\text { length after } \\
\text { each test } t\end{array}$} \\
\hline $\begin{aligned}-60^{\circ} \text { to } \\
-20^{\circ} \mathrm{C} \text { d }\end{aligned}$ & $\begin{array}{l}-20^{\circ} \text { to } \\
+20^{\circ} \mathrm{C}\end{array}$ & $\begin{array}{r}-60^{\circ} \text { to } \\
+200^{\circ} \mathrm{C} \text { 。 }\end{array}$ & $\begin{array}{l}20^{\circ} \text { to } \\
60^{\circ} \mathrm{C}\end{array}$ & $\begin{array}{l}60^{\circ} \text { to } \\
100^{\circ}{ }^{\circ} \mathrm{C}\end{array}$ & $\begin{array}{l}100^{\circ} \text { to } \\
150^{\circ} \mathrm{C}\end{array}$ & $\begin{array}{l}150^{\circ} \text { to } \\
200^{\circ} \mathrm{C}\end{array}$ & $\begin{array}{l}200^{\circ} \text { to } \\
250^{\circ} \mathrm{C}\end{array}$ & $\begin{array}{l}250^{\circ} \text { to } \\
300^{\circ} \mathrm{C}\end{array}$ & $\begin{array}{l}20^{\circ} \text { to } \\
100^{\circ} \mathrm{C}\end{array}$ & $\begin{array}{l}20^{\circ} \text { to } \\
150^{\circ} \mathrm{C}\end{array}$ & $\begin{array}{l}20^{\circ} \text { to } \\
200^{\circ} \mathrm{C}\end{array}$ & $\begin{array}{l}20^{\circ} \text { to } \\
250^{\circ} \mathrm{C}\end{array}$ & $\begin{array}{l}20^{\circ} \text { to } \\
300^{\circ} \mathrm{C}\end{array}$ & \\
\hline$\times 10^{-6}$ & $\times 10^{-6}$ & $\times 10^{-6}$ & $\times 10^{-6}$ & $\times 10^{-6}$ & $\times 10^{-6}$ & $\times 10^{-6}$ & $\times 10^{-6}$ & $\times 10^{-6}$ & $\times 10^{-6}$ & $\times 10^{-6}$ & $\times 10^{-6}$ & $\times 10^{-6}$ & $\times 10^{-6}$ & $\%$ \\
\hline & $\cdots$ & & . & $\cdots$ & $\cdots$ & $\cdots$ & $\cdots$ & - & & ...... & & 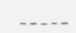 & $\cdots$ & +.001 \\
\hline-.2 & .5 & & & - & $\cdots$ & $\cdots$ & $\cdots$ & $\cdots$ & -... & $\cdots$ & $\cdots$ & …. & 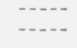 & $\begin{array}{r}+.002 \\
\end{array}$ \\
\hline & .8 & & 1.8 & $\cdots$ & $\cdots$ & $\cdots$ & $\cdots-$ & $\cdots-$ & $\cdots$ & $\cdots$ & $\cdots$ & 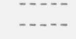 & -...- & .000 \\
\hline & -...-. & & 1.7 & 4.4 & 8.8 & 13.4 & 15.4 & 16.6 & 3.0 & 5.3 & 7.5 & 9.2 & 10.6 & -.001 \\
\hline & $\cdots$ & & 3.2 & 5.8 & 10.5 & 14.0 & 15.8 & 16.4 & 4. 5 & 6.8 & 8.8 & 10.3 & 11.4 & +.002 \\
\hline.-- & -..--- & & 2. 6 & -..- & -..- & $\cdots-\cdot-$ & $\cdots-\cdot$ & $\cdots--$ & -..- & -..- & $\cdots$ & -..- & -..- & -.001 \\
\hline-0 & .8 & .4 & -... & -... & $\cdots$ & $\cdots$ & $\cdots$ & -..-- & $\cdots$ & $\cdots$ & $\cdots$ & $\cdots$ & $\cdots$ & +.001 \\
\hline & & & 2.8 & ... & - o. & $\cdots$ & $\cdots$ & …. & -.... & …. & $\cdots$ & 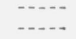 & $\cdots$ & .000 \\
\hline & 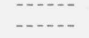 & & 2.7 & 6.0 & 10.1 & 14. 2 & 15.9 & 16.0 & 4.3 & 6.6 & 8.7 & 10.3 & 11. 3 & +.001 \\
\hline$\cdots$ & -..-.. & & $\begin{array}{l}2.1 \\
2.3\end{array}$ & $\cdots$ & -..-. & $\cdots$ & $\cdots$ & $\cdots$ & $\cdots$ & - .... & $\cdots$ & -..... & -.... & -.001 \\
\hline-.1 & 3 & .1 & $\ldots$ & $\cdots$ & $\cdots$ & - & $\cdots$ & $\cdots$ & $\cdots-$ & $\cdots-$ & $\cdots$ & $\cdots$ & $\cdots$ & .000 \\
\hline & -1.e- & & 2. 0 & $\cdots$ & -... & . & -... & - & $\cdots$ & - & - & ... & ..... & .000 \\
\hline & ........ & & $\begin{array}{l}1.2 \\
2.2\end{array}$ & 4.5 & 9.4 & 14.1 & 15.5 & 16.1 & 3.3 & 5.7 & 8.0 & 9.6 & 10.8 & .000 \\
\hline & …... & & $\begin{array}{l}4.6 \\
4.6\end{array}$ & $\begin{array}{l}\text { 8. } 1 \\
8.2\end{array}$ & $\begin{array}{l}12.1 \\
12.3\end{array}$ & $\begin{array}{l}15.2 \\
14.9\end{array}$ & …- & …. & $\begin{array}{l}6.4 \\
6.4\end{array}$ & $\begin{array}{l}8.6 \\
8.7\end{array}$ & $\begin{array}{l}10.4 \\
10.4\end{array}$ & --..- & $\begin{array}{l}12.6 \\
12.6\end{array}$ & $\begin{array}{r}-.001 \\
.000\end{array}$ \\
\hline & $\cdots$ & & 4.5 & -...-. & 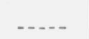 & 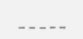 & 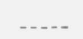 & 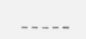 & - & ..... & 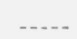 & 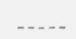 & 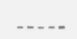 & .000 \\
\hline .0 & 1. 8 & .9 & & $\cdots$ & …- & $\cdots$ & -.... & …. & $\cdots$ & -..-.. & $\cdots$ & 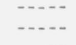 & -...- & +.001 \\
\hline & $\begin{array}{r}1.9 \\
-\end{array}$ & & 4.5 & -...- & …-. & $\ldots$ & -..-- & N-... & ;...- & -...- & - . - & -..-. & …- & .000 \\
\hline & -..... & & $\begin{array}{l}\text { t.5 } \\
4.5\end{array}$ & 8.0 & 12.3 & 14. 9 & 15.9 & 16.3 & 6.3 & 8.6 & 10.3 & 11.6 & 12.4 & .000 \\
\hline & -.--.- & & $\begin{array}{l}3.8 \\
3.8\end{array}$ & $\cdots-$ & -..-. & -.-- & --.-. & --.- & -..-. & $\cdots-\cdot$ & -.-. & $\cdots$ & -...- & .000 \\
\hline $\begin{array}{r}3 \\
3 \\
3\end{array}$ & $\begin{array}{l}1.2 \\
1.3\end{array}$ & .7 & & $\cdots$ & $\cdots$ & $\cdots$ & $\cdots$ & $\cdots$ & $\cdots$ & $\cdots$ & $\cdots$ & $\cdots$ & $\cdots$ & .000 \\
\hline & & & $\begin{array}{l}3.6 \\
3.8\end{array}$ & -..-. & -... & -.... & …- & 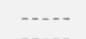 & -...- & -...- & -...- & -.... & …-. & -.001 \\
\hline 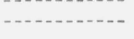 & 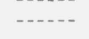 & $\cdots$ & 3.6 & 6.8 & 11.2 & 14.4 & -..- & …- & 5.2 & 7.5 & 9.4 & $\cdots$ & 11.8 & .000 \\
\hline & -..-. & & 73 & -..- & -..- & $\cdots$ & -...- & -...- & -...- & $\cdots$ & -..-- & -... & $\cdots$ & .000 \\
\hline $\begin{array}{l}1.5 \\
1.5\end{array}$ & $\begin{array}{l}4.0 \\
4.0\end{array}$ & $\begin{array}{l}2.8 \\
2.8\end{array}$ & $\ldots$ & -.... & -.... & -.... & …. & …. & - & -...- & -.... & -..... & -.... & .000 \\
\hline - & -...... & --.-- & 6.4 & 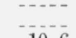 & $\cdots$ & - & 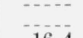 & 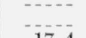 & $\cdots$ & - & - & $\cdots$ & 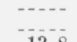 & .000 \\
\hline & & & & & 10. & 10.4 & 10.4 & 16.4 & 9.1 & 10.9 & 12.1 & 10.0 & 10.8 & -.002 \\
\hline
\end{tabular}

\section{Results and Discussion}

Determinations of linear thermal expansion of the samples of cobalt-iron-chromium alloys were made at various temperatures between $-65^{\circ}$ and $+950^{\circ} \mathrm{C}$ and of the effects of various heat treatments (from $-196^{\circ}$ to $+1,000^{\circ} \mathrm{C}$ ) on their thermal expansion. The results are summarized in 3 tables and 8 figures.

Table 1 gives the coefficients of expansion that were determined for various temperature ranges from $-60^{\circ}$ to $+300^{\circ} \mathrm{C}$. The phase of the samples for each test and the residual change in length after each test are also given in table 1 .

Figure 1 shows expansion curves of nine samples of cobalt-iron-chromium alloys between $-65^{\circ}$ and $+60^{\circ}$ C. These samples are arranged, from the bottom to the top, in increasing values of their coefficients of expansion for the range from $+20^{\circ}$ to $+60^{\circ}$ C. For all of the samples that were investigated between $-65^{\circ}$ and $+60^{\circ} \mathrm{C}$ the observations were made in the following order: (a) Heating and cooling between room temperature and $+60^{\circ} \mathrm{C}$ (test 1); (b) cooling and heating between room tem- perature and $-65^{\circ} \mathrm{C}$ (test 2); (c) again heating and cooling between room termperature and $+60^{\circ} \mathrm{C}$ (test 3). Several of the samples in figure 1 have coefficients of expansion that are very nearly zero for the range from $-65^{\circ}$ to $+20^{\circ} \mathrm{C}$.

Figure 2 shows the expansion of a low-expansion alloy over the range from $-65^{\circ}$ io $+300^{\circ} \mathrm{C}$. While the average coefficient of expansion of this sample is less than $1 \times 10^{-6} / \mathrm{deg} \mathrm{C}$ over the range from $-65^{\circ}$ to $+60^{\circ} \mathrm{C}$, it rapidly increases to $15.0 \times 10^{-6} / \mathrm{deg} \mathrm{C}$ over the range from $200^{\circ}$ to $300^{\circ} \mathrm{C}$. It is seen that the low coefficients of expansion for such alloys generally apply for temperatures between $-65^{\circ}$ and $+60^{\circ} \mathrm{C}$.

Figure 3 indicates graphically the relations between chemical composition and the average coefficients of expansion of the samples in the first tests for the range from $20^{\circ}$ to $60^{\circ} \mathrm{C}$. All of the coefficients of expansion apply for the samples in the original annealed conditions ( $\gamma$ phase). The curve in this figure represents an isodil ${ }^{3}$ equal to $1.0 \times$

3 The word "isodil" was derived in 1931 by Hidnert, from "iso" (Greek isos, meaning equal) and from the first three letters of "dilatation", and denotes a meaning equal) and from the first three letters of "dilat
curve representing compositions having equal expansion. 

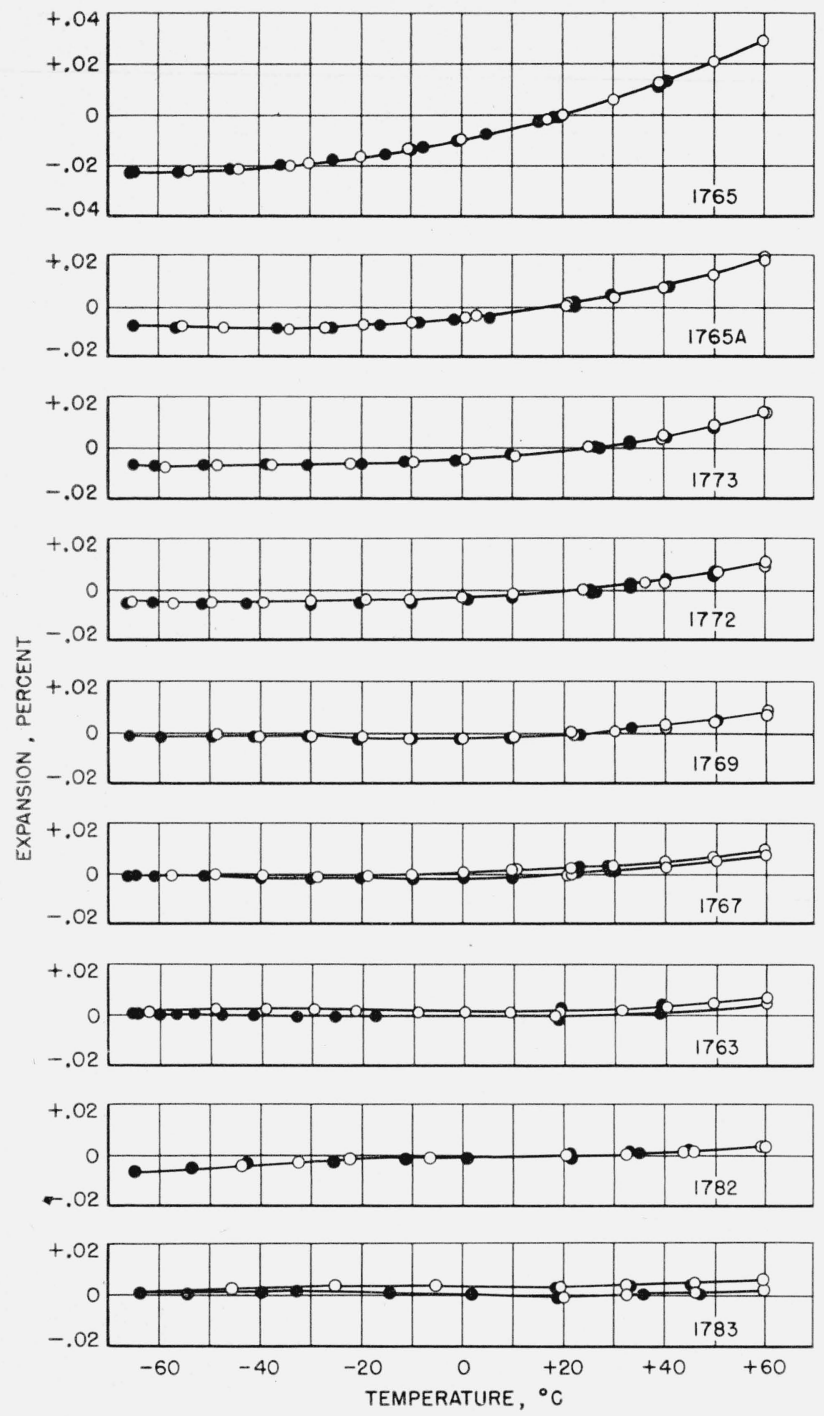

FIGURE 1. Linear thermal expansion of nine annealed cobaltiron-chromium alloys (Fe 36.2 $2_{2}$ to $36.9_{2}$, $\mathrm{Cr} 9.0_{9}$ to $9.8_{7} \%$ ). The initial observation for each alloy was taken at about $20^{\circ} \mathrm{C}$ and is plotted on the zero ordinate.

$\bigcirc$, Heating; 0 , cooling.

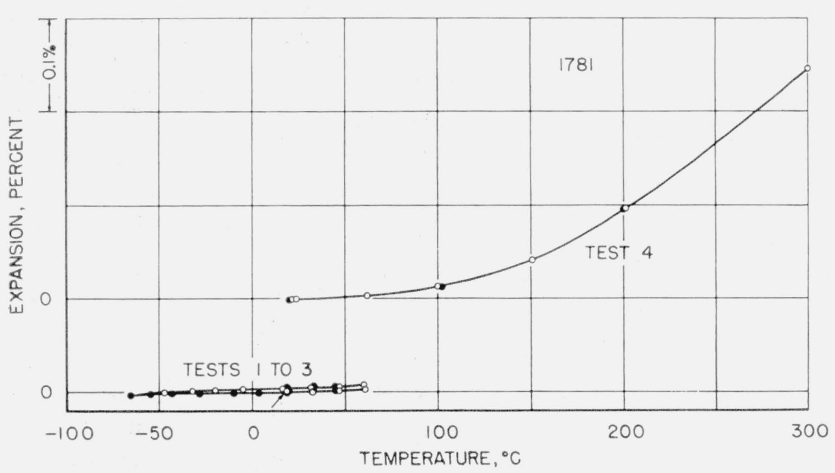

Figure 2. Linear thermal expansion of annealed cobalt-ironchromium alloy (Fe 36.5, $\mathrm{Cr} 9.0_{6} \%$ ).

$\bigcirc$, Heating; , cooling; $\rightarrow$, initial observation.

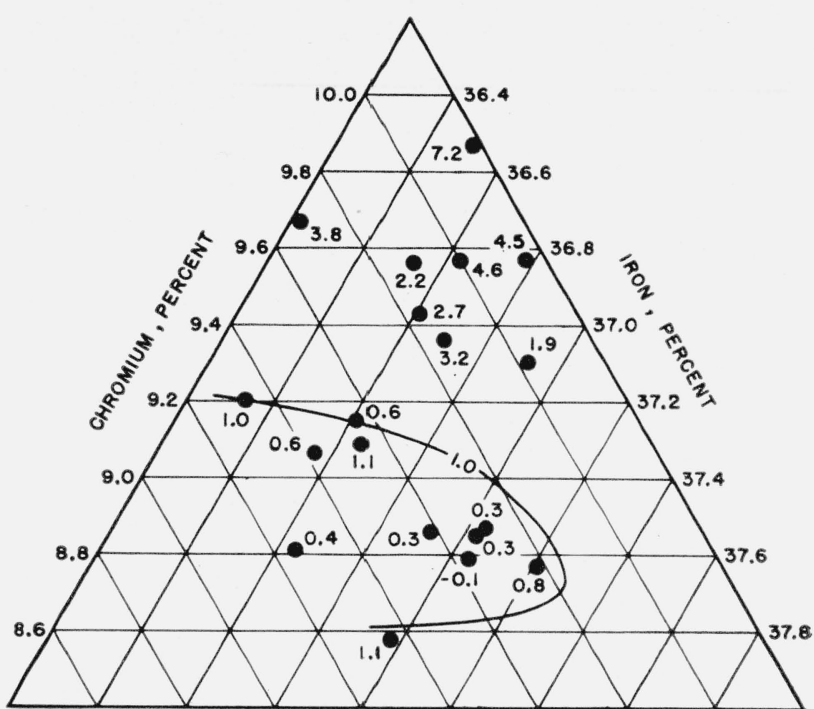

$\longleftarrow$ COBALT

Figure 3. Portion of ternary diagram indicating the effects of composition (percentage by weight) on the coefficients of linear expansion (in millionths per degree $C$ ) of annealed cobaltiron-chromium alloys for the range $20^{\circ}$ to $60^{\circ} \mathrm{C}$.

$10^{-6} / \mathrm{deg} \mathrm{C}$, and appears to approximate part of an ellipse as shown by Masumoto [1]. The results indicate that the low-expanding alloys lie within this elliptical-shaped isodil and that the alloys outside of it have coefficients that generally increase with increasing distance from it. These results indicate that close chemical control is necessary in the preparation of these low-expanding alloys.

The samples with chromium contents between $8.5_{6}$ to $9.1_{5}$ percent have very low coefficients of expansion at room temperature but $\gamma \rightarrow \alpha$ transformations started between $-10^{\circ}$ and $-80^{\circ} \mathrm{C}$. $\mathrm{Ar}_{3}$ temperatures for five samples may be found in table 1 (see also footnote q). These values indicate that for the samples investigated the $\mathrm{Ar}_{3}$ temperature tends to decrease as the chromium content increases. No $\gamma \rightarrow \alpha$ transformation was found on cooling a sample (see table 1, footnote r) containing $9.5_{7}$ percent chromium, to as low as $-196^{\circ} \mathrm{C}$. Masumoto [1] reported that his low-expanding alloys showed no $\gamma \rightarrow \alpha$ transformations even when cooled to liquid hydrogen temperature $\left(-253^{\circ} \mathrm{C}\right)$, but four of his alloys expanded slightly (less than 0.02 percent) on cooling to low temperatures.

Figure 4 shows the $\gamma \rightarrow \alpha$ transformation that occurred on cooling sample 1762 to $-67^{\circ} \mathrm{C}$. This transformation was not completed after reaching this low temperature. The expansion on cooling caused by the $\gamma \rightarrow \alpha$ transformation proceeded with discrete rapid increases in length. These discrete increases in length were accompanied by audible clicks and slight increases in temperature of the sample. Upon heating the sample from $-67^{\circ} \mathrm{C}$. it was found that the rate of expansion was larger than it had been for the sample in its original annealed condition. It was found that for temperatures below $200^{\circ} \mathrm{C}$ the rate of expansion increases with increasing amounts of $\alpha$ phase. 


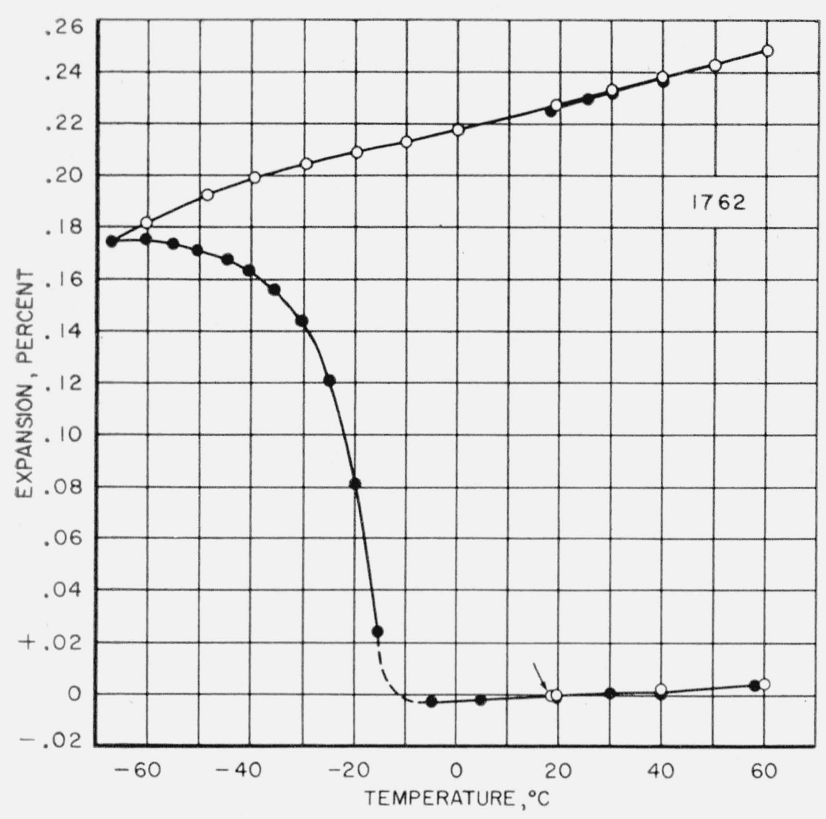

Figure 4. Linear thermal expansion curve of annealed cobaltiron-chromium alloy (Fe 36.98, $\mathrm{Cr} 8.5_{6} \%$ ) showing $\gamma \rightarrow \alpha$ transformation on cooling.

$\mathrm{O}$, Heating; •, cooling; $\rightarrow$, initial observation.

The $\gamma \rightarrow \alpha$ transformation in sample $1766 \mathrm{~A}$ (cut from same rod as sample 1766) proceeded isothermally when its temperature was maintained at $-70^{\circ} \mathrm{C}$ for 3 days. On rapid cooling from room temperature to $-70^{\circ} \mathrm{C}$, the sample expanded 0.12 percent. Its length at $-70^{\circ} \mathrm{C}$ increased with time at a diminishing rate. At the end of 3 days, the additional increase at $-70^{\circ} \mathrm{C}$ was 0.06 percent.

Figure 5 also shows an incomplete $\gamma \rightarrow \alpha$ transformation that occurred in sample 1758 on cooling to $-64^{\circ} \mathrm{C}$. Although the sample was cooled to $-80^{\circ}$ $\mathrm{C}$ before the fifth test, the rate of expansion during the fifth test was practically the same as the rate during the third and fourth tests. This indicates that little or no additional $\gamma \rightarrow \alpha$ transformation occurred during cooling between $-64^{\circ}$ and $-80^{\circ} \mathrm{C}$. The rate of expansion during the sixth test after cooling to $-196^{\circ} \mathrm{C}$, indicates that an additional $\gamma \rightarrow \alpha$ transformation occurred in the sample. After the sixth test the sample was given a prolonged annealing at high temperatures (see table 1, footnote k) and then cooled slowly to room temperature. The smaller rate of expansion in the seventh test as compared to the rate in the sixth test of the sample, indicates that the annealing treatment resulted in a partial transformation of the $\alpha$ to the $\gamma$ condition. Reference should also be made to the coefficients of expansion (between $20^{\circ}$ and $60^{\circ} \mathrm{C}$ ) in table 1 for a better comprehension of the comparisons between the tests indicated in figure 5.

Figure 6 shows the results obtained on six tests of sample 1760 . The first three tests were made with the sample in the original $\gamma$ condition. The third test did not show any irregularities to $700^{\circ} \mathrm{C}$. After the third test, the sample was cooled rapidly to

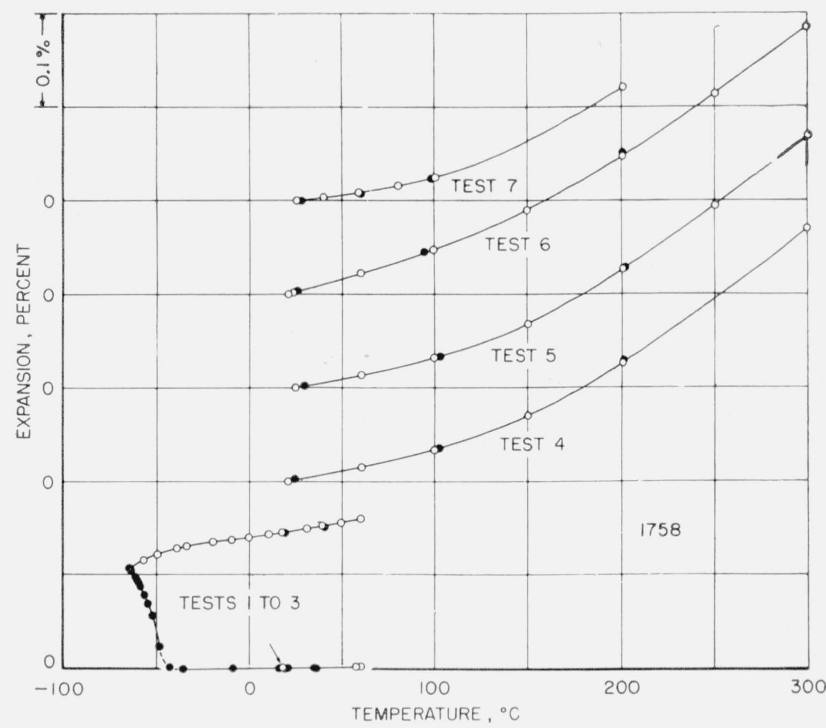

FiguRE 5. Linear thermal expansion of annerled cobalt-ironchromium alloy (Fe $36.9_{2}$, Cr 8.86\%).

Combined tests 1 to 3 show $\gamma \rightarrow \alpha$ transformation on cooling; before fifth test, sample was cooled to $-80^{\circ} \mathrm{C}$; before sixth test, sample was cooled to $-196^{\circ} \mathrm{C}$; before seventh test, sample was hydrogen annealed at $850^{\circ} \mathrm{C}$ for $10 \mathrm{hr}$ and at $1,000^{\circ} \mathrm{C}$ for $1 \mathrm{hr}$, and furnace cooled in $22 \mathrm{hr}$.

, Heating; , cooling; $\rightarrow$, initial observation.

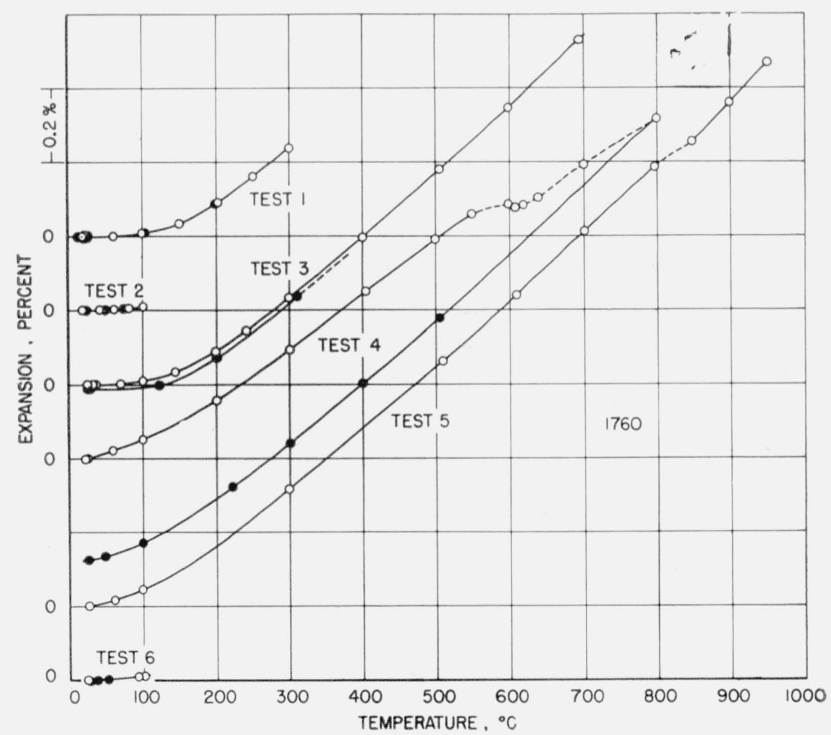

Figure 6. Linear thermal expansion of annealed cobalt-ironchromium alloy ( $\mathrm{Fe} 37.0_{4}$, Cr $8.8_{7} \%$ ).

Before fourth test, sample was cooled to $-196^{\circ} \mathrm{C}$.

$O$, Heating; $\boldsymbol{\theta}$, cooling.

$-190^{\circ} \mathrm{C}$ and held at this temperature several hours. A comparison of the expansion curves on heating in the third and fourth tests indicates that the cooling to $-190^{\circ} \mathrm{C}$ transformed a part or all of the $\gamma$ to $\alpha$. An examination of the data in this paper indicates that the $\gamma \rightarrow \alpha$ transformation in this sample was the most complete of any that were investigated in this work. On heating the sample in the fourth test, an $\alpha \rightarrow \gamma$ transformation ${ }^{4}$ started at about $600^{\circ} \mathrm{C}$.

${ }^{4}$ Confirmed with other samples. 
TaBle 2. Coefficients of linear thermal expansion of cobalt-iron-chromium alloy (sample 1760; Fe $37.04, \mathrm{Cr} 8.87 \%$ )

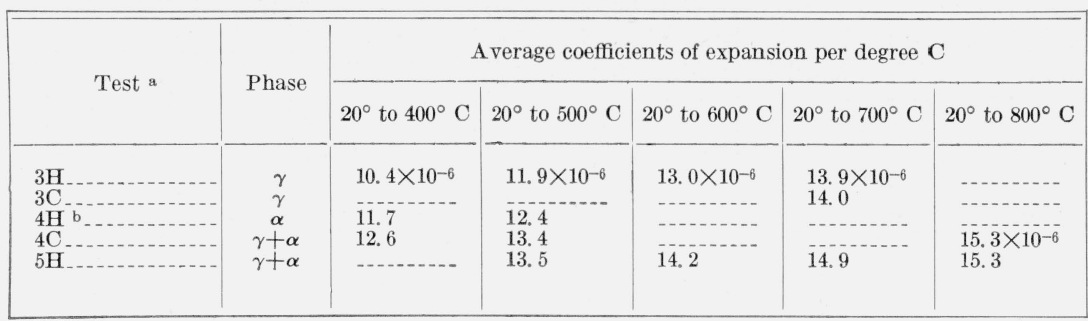

a $\mathrm{H}$ indicates that the coefficients of expansion were obtained on heating and $\mathrm{C}$ indicates that they were obtained on cooling.

b Before this test, cooled to $-190^{\circ} \mathrm{C}$,

The $\alpha \rightarrow \gamma$ transformation was completed during heating to $950^{\circ} \mathrm{C}$ in the fifth test, as indicated by the low expansion in the sixth test (compare with results of tests 1 to 3 for the $\gamma$ condition).

Table 2 gives the average coefficients of expansion of sample 1760 for various temperature ranges from $20^{\circ}$ to $800^{\circ} \mathrm{C}$. Figure 7 shows the coefficients of expansion versus temperature for the $\gamma$ and $\alpha$ phases of this sample. The marked change in slope of the curve for the sample in the $\gamma$ condition at about $240^{\circ} \mathrm{C}$ is typical for these alloys and is apparently associated with the magnetic transformation at the Curie point. ${ }^{5}$ For various mixtures of $\alpha$ and $\gamma$ in this alloy, the corresponding curves for the coefficients of expansion versus temperature generally lie between the curves shown for $\alpha$ and $\gamma$ (e. g., table 1, sample 1760).

Figure 8 reproduced from Köster [7] indicates that the boundary between $\alpha$ and $\gamma$ phases is a steeply ascending curve up to $600^{\circ} \mathrm{C}$ in the region of 9 percent of chromium. The conclusion, deduced from the data reported in table 1 of the present investigation, that the $\mathrm{Ar}_{3}$ temperatures of the alloys decrease as the chromium content increases, confirms this equilibrium diagram. The $A c_{1}$ temperatures obtained on three samples at about $600^{\circ} \mathrm{C}$ are also in agreement with this diagram. An examination of this figure in conjunction with data in table 1 also indicates that $\alpha$ phase should not be found in these alloys containing more than 9.6 percent chromium.

\footnotetext{
5 The authors found that a specimen cut from sample 1757 in the $\gamma$ condition The authors found that a specimen cut from sample 1757 in the $\gamma$ condition lost its magnetization at about $240^{\circ} \mathrm{C}$. Masumoto [1] indicated that the mag-
netic transformation point of low-expanding cobalt-iron-chromium alloys is in netic transformation
the vicinity of $250^{\circ} \mathrm{C}$.
}

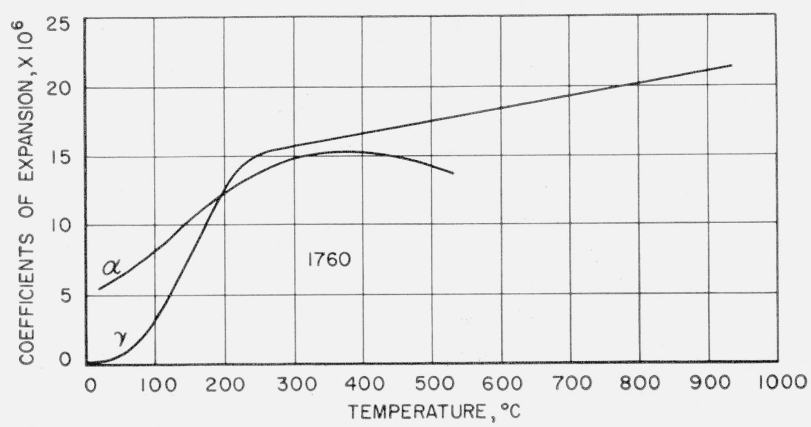

FIGURE 7. Variation of coefficients of expansion versus temperature for $\gamma$ and $\alpha$ phases of a cobalt-iron-chromium alloy (Fe $37.0_{4}, \mathrm{Cr} 8.87 \%$ ).
The coefficients of expansion in tables 1 and 2 indicate the presence of $\alpha$ and $\gamma$ phases in the samples due to various heat treatments. Another indication of their presence is the residual change in length at $20^{\circ} \mathrm{C}$. Table 3 gives the changes in length at $20^{\circ} \mathrm{C}$ from the $\gamma$ condition of five samples after various heat treatments. The amount of $\alpha$ phase present in a sample appears to be approximately proportional to its increase in length.

TABLE 3. Changes in length caused by transformations in 5 samples of cobalt-iron-chromium alloys

\begin{tabular}{|c|c|c|c|c|c|}
\hline \multirow{2}{*}{ Heat treatment } & \multicolumn{5}{|c|}{$\begin{array}{c}\text { Cumulative changes a in length (at } 20^{\circ} \mathrm{C} \text { ) after } \\
\text { successive heat treatments }\end{array}$} \\
\hline & 1757 & 1758 & 1760 & $1766 \mathrm{~A} \mathrm{~b}$ & $1783 \mathrm{~A}$ \\
\hline As received ( $\gamma$ condi- & $\%$ & $\%$ & $\%$ & $\%$ & $\%$ \\
\hline $\begin{array}{l}\text { tion) } \\
\text { Cooled to }-65^{\circ} \mathrm{C}\end{array}$ & $\begin{array}{r}0.000 \\
+\quad 170\end{array}$ & $\begin{array}{r}0.000 \\
+\quad 146\end{array}$ & 0.000 & 0.000 & 0.000 \\
\hline Cooled to $-80^{\circ} \mathrm{C}$ & $\begin{array}{r}+.170 \\
.173\end{array}$ & $\begin{array}{r}+.146 \\
.150\end{array}$ & (n) & (n) & (n- \\
\hline Cooled to $-190^{\circ} \mathrm{C}_{-}$ & & -100 & +.265 & & \\
\hline Cooled to $-196^{\circ} \mathrm{C}$ & .179 & .263 & - n- & +.243 & +.182 \\
\hline Heated to $+700^{\circ} \mathrm{C}$ & $-\ldots+$ & - & - & .067 & .070 \\
\hline Cooled to $-196^{\circ} \mathrm{C}$ & -..... & (n) & & .078 & .070 \\
\hline Heated to $1,000^{\circ} \mathrm{C}$.- & .069 & .068 & - n & .006 & -.001 \\
\hline $\begin{array}{l}\text { Held at }-70^{\circ} \mathrm{C} \text { for } \\
3 \text { days }\end{array}$ & & & & .230 & \\
\hline Cooled to $-196^{\circ} \mathrm{C}_{-}$ & & & & .232 & +.182 \\
\hline
\end{tabular}

\& Where values are not indicated, the heat treatments were not given. b Cut from same rod as sample 1766 .

- Cut from same rod as sample 1783 .

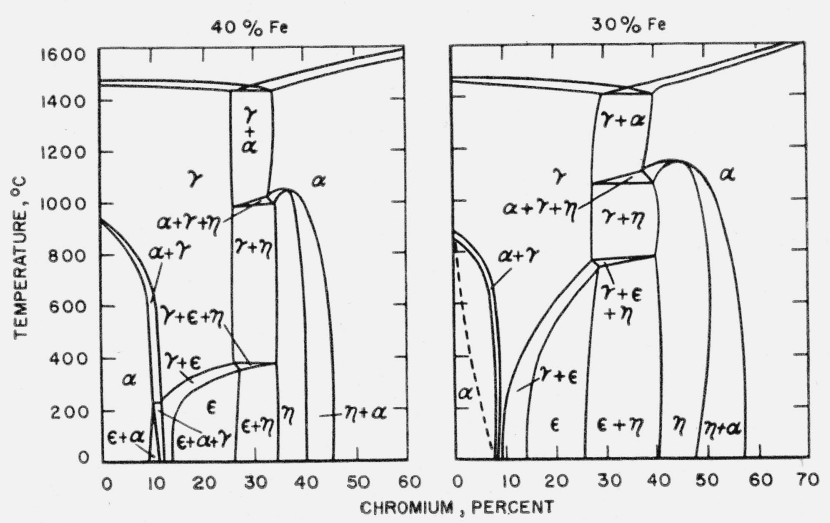

FIGURE 8. Two sections of ternary equilibrium diagram of cobali-iron-chromium alloys for 40 and 30 percent iron (Köster [7]). 

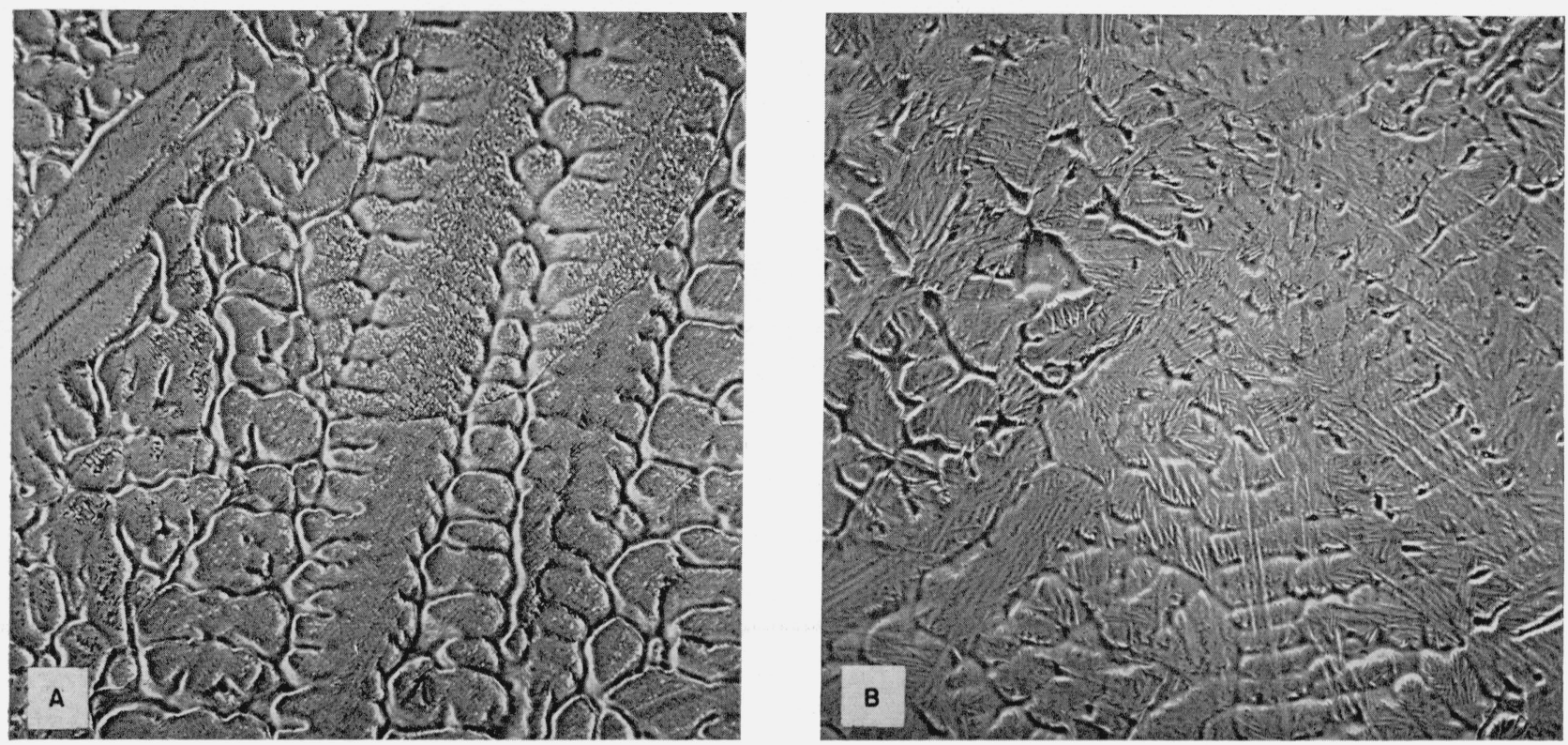

Figure 9. Microstructure $(\times 75)$ of two cobalt-iron-chromium alloys.

Etched with hydrochloric and picric acids in alcohol [10]. A, Sample 1766, after annealing at $1,000^{\circ} \mathrm{C}$; B, sample 1757 , after cooling to $-196^{\circ} \mathrm{C}$.

A metallographic examination ${ }^{6}$ was made of some of the samples in the $\gamma$ and $\alpha+\gamma$ conditions. Typical structures are shown in the photomicrographs of figure 9. This figure shows that the annealed sample (fig. $9 \mathrm{~A}, \gamma$ ) has large grains and dendrites whereas the sample that had been cooled to $-196^{\circ} \mathrm{C}$ (fig. 9B, $\alpha+\gamma$ ) has a needle-like structure. The needle-like structure formed during the transforma. tion on cooling and the kinetics of the transformation indicated by the dilatometric experiments, shows that this transformation is of the martensitic type $[8,9]$.

\section{Summary}

1. The linear thermal expansion and phase transformations of some cobalt-iron-chromium alloys were investigated at various termperatures between $-65^{\circ}$ and $+950^{\circ} \mathrm{C}$.

2. Annealed alloys having chemical compositions of about 54 percent cobalt, 36.6 percent iron, and 8.9 percent chromium have coefficients of expansion less than $1 \times 10^{-6} / \mathrm{deg} \mathrm{C}$ for the range from $20^{\circ}$ to $60^{\circ}$ C. Close control of chemical composition is a prerequisite for success in the production of lowexpanding cobalt-iron-chromium alloys.

3. For temperatures above $60^{\circ} \mathrm{C}$, the coefficients of expansion of the alloys increase to about $16 \times 10^{-6} /$ $\operatorname{deg} \mathrm{C}$ for the range from $250^{\circ}$ to $300^{\circ} \mathrm{C}$.

4. Some low-expanding alloys were found to have a $\gamma \rightarrow \alpha$ transformation on cooling to low temperatures. This transformation is of the martensitic type in that it produced a needle-like structure and

${ }^{6}$ By D. B. Ballard of the Metallurgy Division of the National Bureau of Standards. proceeded with discrete rapid increases in length, accompanied by audible clicks and slight increases in temperature.

5. The coefficients of expansion below $200^{\circ} \mathrm{C}$ increase with increasing amounts of $\alpha$ phase present in the alloys.

6. Samples containing $\alpha$ started to transform to $\gamma$ at about $600^{\circ} \mathrm{C}$.

\section{References}

[1] H. Masumoto, On the thermal expansion of alloys of cobalt, iron, and chromium, and a new alloy, "Stainless-Invar", Science Reports Tôhoku Univ. 23, 265 (1934).

[2] Private communication from H. Masumoto.

[3] W. Souder and P. Hidnert, Measurements on the thermal expansion of fused silica, Sci. Pap. BS 21, 1 (192627) 5524.

[4] Invar and related nickel steels (2d edition), Circular BS No. 58 (1923).

[5] H. Masumoto and H. Saito, On Young's modulus and its temperature coefficient of the alloys of cobalt, iron and chromium, and a new alloy "Co-elinvar", Science Reports Research Institutes, Tôhoku Univ. A1, 17 (1949).

[6] P. Hidnert and W. Souder, Thermal expansion of solids, NBS Circular 486 (1950).

[7] W. Köster, Das System Eisen-Kobalt-Chrom, Archiv Eisenhüttenwesen 6, 113 (1932).

[8] A. R. Troiano and A. B. Greninger, The martensite transformation, Metal Progress 50, 303 (1946) or Metals Handbook, Amer. Soc. Metals, p. 263 (1948).

[9] M. Cohen, The martensite transformation, Symposium on phase transformations in solids, p. 588 (John Wiley \& Sons, Inc., New York, N. Y., 1951).

[10] Subcommittee on etching reagents for iron and steel, Etching reagents for the microscopic examination of iron and steel, Metals Handbook, Amer. Soc. Metals, p. 394 (1948).

Washington, December 27, 1954. 